\title{
Naturwissenschaftliche, ethische und rechtliche Empfehlungen zur klinischen Translation der Forschung mit humanen induzierten pluripotenten Stammzellen und davon abgeleiteten Produkten
}

\author{
Sara Gerke, Solveig Lena Hansen, Verena Christine Blum, Stephanie Bur, \\ Clemens Heyder, Christian Kopetzki, Ina Meiser, Julia C. Neubauer, \\ Danielle Noe, Claudia Steinböck, Claudia Wiesemann, Heiko Zimmermann \\ und Jochen Taupitz
}

Zusammenfassung In der öffentlichen Debatte wurden humane induzierte pluripotente Stammzellen (hiPS-Zellen) im Vergleich zu humanen embryonalen Stammzellen (hES-Zellen) oft als moralisch unproblematische Alternative zur verbrauchenden Forschung mit Embryonen dargestellt. Doch bei genauerer Betrachtung wird klar, dass insbesondere die klinische Translation der Forschung mit hiPS-Zellen und davon abgeleiteten Produkten eigene ethische, aber auch naturwissenschaftliche und rechtliche Probleme aufwirft. Im Jahr 2016 hat die International Society for Stem Cell Research (ISSCR) Leitlinien für die klinische Translation der Stammzell-

Die Originalversion dieses Kapitels wurde korrigiert. Ein Erratum finden Sie unter https://doi.org/10.1007/978-3-662-59052-2_12

\footnotetext{
S. Gerke $(\bowtie)$

The Petrie-Flom Center for Health Law Policy, Biotechnology, and Bioethics at Harvard Law School, Harvard University, Cambridge, USA

E-Mail: sgerke@1aw.harvard.edu

S. L. Hansen · C. Heyder · C. Wiesemann

Institut für Ethik und Geschichte der Medizin, Universitätsmedizin Göttingen,

Göttingen, Deutschland

E-Mail: solveig-lena.hansen@medizin.uni-goettingen.de; clemens.heyder@medizin. uni-goettingen.de; cwiesem@gwdg.de

V. C. Blum · D. Noe · C. Steinböck

Abteilung Medizinrecht des Instituts für Staats- und Verwaltungsrecht der Universität Wien, Wien, Österreich

E-Mail: verena.blum@univie.ac.at; danielle.monika.noe@univie.ac.at; claudia.steinboeck@ univie.ac.at
}

S. Gerke et al. (Hrsg.), Die klinische Anwendung von humanen induzierten pluripotenten Stammzellen, Veröffentlichungen des Instituts für Deutsches, Europäisches und Internationales Medizinrecht, Gesundheitsrecht und Bioethik der Universitäten Heidelberg und Mannheim 48, https://doi.org/10.1007/978-3-662-59052-2_11 
forschung veröffentlicht. Diese Leitlinien beziehen sich allerdings nicht exklusiv auf hiPS-Zellen und berücksichtigen ferner nicht die Perspektiven aller beteiligten Stakeholder. Für die Ausgestaltung der klinischen Translation der hiPS-ZellForschung ist sowohl die Schaffung geeigneter naturwissenschaftlicher und rechtlicher Rahmenbedingungen als auch angemessener ethischer Vorgaben dringend erforderlich. Der durch das Bundesministerium für Bildung und Forschung geförderte deutsch-österreichische Forschungsverbund „ClinhiPS“ entwickelte zu diesem Zweck die vorliegenden naturwissenschaftlichen, ethischen und rechtlichen Empfehlungen zur klinischen Translation der Forschung mit hiPS-Zellen und davon abgeleiteten Produkten.

Seit einigen Jahren ist es möglich, humane induzierte pluripotente Stammzellen (hiPS-Zellen) im Labor zu erzeugen. Für die Entdeckung, dass reife, spezialisierte Zellen zu pluripotenten Stammzellen reprogrammiert werden können, wurden Shinya Yamanaka und John B. Gurdon 2012 mit dem Nobelpreis ausgezeichnet. HiPS-Zellen können mit Hilfe spezifischer genetischer Faktoren aus nahezu jeder Zelle des Menschen erzeugt werden und erlangen dadurch wieder Fähigkeiten, die eigentlich nur Zellen eines frühen Embryos besitzen: Sie sind unbegrenzt teilungsfähig und können sich in alle Zellen des menschlichen Körpers entwickeln (differenzieren). Die Forschung auf diesem Gebiet hat in den letzten Jahren große Fortschritte gemacht, weshalb erste klinische Anwendungen in greifbare Nähe rücken. Es wird etwa diskutiert, hiPS-Zellen für zukünftige Zelltherapien zu nutzen, um nach zellzerstörenden Krankheiten oder Unfällen, beispielsweise nach Herzinfarkt oder Verbrennungen, Ersatz zu schaffen.

In der öffentlichen Debatte wurde dieser Zelltyp im Vergleich zu humanen embryonalen Stammzellen (hES-Zellen) oft als moralisch unproblematische Alternative zur verbrauchenden Forschung mit Embryonen dargestellt. ${ }^{1}$ Doch bei genauerer Betrachtung wird klar, dass insbesondere die klinische Translation der Forschung mit hiPS-Zellen und davon abgeleiteten Produkten eigene ethische, aber auch eigene naturwissenschaftliche und rechtliche Probleme aufwirft. Sie werden durch

\footnotetext{
${ }^{1}$ www.ClinhiPS.de. ISSCR 2016.
}

\section{Kopetzki}

Institut für Staats- und Verwaltungsrecht, Universität Wien, Wien, Österreich

E-Mail: christian.kopetzki@univie.ac.at

S. Bur · I. Meiser

Fraunhofer-Institut für Biomedizinische Technik IBMT, Sulzbach, Deutschland

E-Mail: stephanie.bur@gmx.de; ina.meiser@ibmt.fraunhofer.de

J. C. Neubauer

Fraunhofer-Projektzentrum für Stammzellprozesstechnik, Würzburg, Deutschland

E-Mail: julia.neubauer@ibmt.fraunhofer.de

H. Zimmermann

Fraunhofer-Institut für Biomedizinische Technik, Sulzbach, Deutschland

E-Mail: Heiko.Zimmermann@ibmt.fraunhofer.de

J. Taupitz

IMGB, Universität Mannheim, Mannheim, Deutschland

E-Mail: taupitz@jura.uni-mannheim.de 
die derzeitige Regelung der klinischen Forschung nur unzureichend erfasst. Im Jahr 2016 hat die International Society for Stem Cell Research (ISSCR) zwar Leitlinien für die klinische Translation der Stammzellforschung veröffentlicht. ${ }^{2}$ Diese Leitlinien beziehen sich allerdings nicht exklusiv auf hiPS-Zellen und berücksichtigen zudem nicht die Perspektiven aller beteiligten Stakeholder. Für die Ausgestaltung der klinischen Translation der hiPS-Zell-Forschung ist deshalb sowohl die Schaffung geeigneter naturwissenschaftlicher und rechtlicher Rahmenbedingungen als auch angemessener ethischer Vorgaben dringend erforderlich.

$\mathrm{Zu}$ diesem Zweck wurde im Rahmen einer Förderung durch das deutsche Bundesministerium für Bildung und Forschung der Forschungsverbund „ClinhiPS“ von deutschen und österreichischen Wissenschaftlerinnen und Wissenschaftlern ${ }^{3}$ aus Naturwissenschaft, Medizinethik und Recht gegründet. Im Rahmen dieses Verbunds wurden in zweijähriger gemeinsamer Arbeit die spezifischen mit der klinischen Translation von hiPS-Zellen und davon abgeleiteten Produkten verbundenen Probleme erfasst und analysiert. Des Weiteren wurden die naturwissenschaftlichen Herausforderungen für Qualität und Sicherheit im Kontext der klinischen Anwendung am Menschen untersucht sowie aktuelle Lücken in der ethischen und rechtlichen Regulierung identifiziert.

Der Forschungsverbund „ClinhiPS“ entwickelte darauf aufbauend die vorliegenden naturwissenschaftlichen, ethischen und rechtlichen Empfehlungen zur klinischen Translation der Forschung mit hiPS-Zellen und davon abgeleiteten Produkten (Abb. 1 und 2).

\section{Einleitung}

Diese Empfehlungen beziehen sich auf den Prozess der Entwicklung und Erprobung hiPS-Zell-basierter Produkte sowie deren klinische Anwendung am Menschen.

HiPS-Zell-basierte Produkte sind Produkte, die auf der Grundlage von hiPSZellen und ihren Differenzierungsderivaten hergestellt werden. HiPS-Zellen werden aus menschlichen Körperzellen (z. B. Haut- oder Blutzellen) hergestellt. Sie besitzen das Potenzial, sich in alle Zelltypen des menschlichen Körpers (z. B. Herzmuskel-, Gehirn- oder Nierenzellen) zu entwickeln. ${ }^{4}$

HiPS-Zellen sind bereits heute ein wertvolles Tool für die Testung von Arzneimitteln und die Entwicklung von Krankheitsmodellen im Labor. ${ }^{5}$ Sie sollen in Zukunft auch für die klinische Anwendung genutzt werden und so vor allem zur besseren Behandlung von Krankheiten beitragen. ${ }^{6}$

Die Empfehlungen richten sich an alle Stakeholder, die in die klinische Translation der hiPS-Zell-Forschung, also die Übertragung von Ergebnissen der (Grundlagen-)Forschung in die klinische Praxis, involviert sind. Als Stakeholder werden Individuen oder Gruppen verstanden, die an der Entwicklung und Anwendung hiPS-Zell-basierter Produkte beteiligt oder von ihr betroffen sind. Hierzu zählen ins-

\footnotetext{
${ }^{2}$ ISSCR 2016.

${ }^{3} \mathrm{Im}$ Folgenden wird die maskuline Form aus Gründen der leichteren Lesbarkeit verwendet.

${ }^{4}$ www.ClinhiPS.de. Zugegriffen: 12.10.2019.

${ }^{5}$ www.ClinhiPS.de. Zugegriffen: 12.10.2019.

${ }^{6}$ www.ClinhiPS.de. Zugegriffen: 12.10.2019.
} 
EINLEITUNG

\begin{tabular}{|c|c|c|c|}
\hline \multicolumn{4}{|c|}{ 1. NATURWISSENSCHAFTLICHE ASPEKTE } \\
\hline 1.1 & Allgemeines & 1.3 & Expansion von hiPS-Zellen \\
\hline 1.1.1: & $\begin{array}{l}\text { Anwendung von Good Manufacturing } \\
\text { Practice }\end{array}$ & $\begin{array}{l}\text { 1.3.1: } \\
1.3 .2:\end{array}$ & $\begin{array}{l}\text { Auswahl des Expansionssystems } \\
\text { Automatisierung }\end{array}$ \\
\hline 1.1.2: & $\begin{array}{l}\text { GMP-konforme Herstellung von Medien } \\
\text { und Reagenzien }\end{array}$ & 1.3.3: & Qualitätskontrollen \\
\hline 1.1.3: & Datenintegration & 1.4 & Differenzierung von hiPS-Zellen \\
\hline $\begin{array}{l}\text { 1.1.4: } \\
\text { 1.1.5: }\end{array}$ & $\begin{array}{l}\text { Identifikation von Langzeitrisiken } \\
\text { Standardisierte Qualitätskontrollen }\end{array}$ & 1.4.1: & Differenzierungsfaktoren \\
\hline 1.1.6: & $\begin{array}{l}\text { Implementierung zellbiologischer } \\
\text { Sicherheitsmechanismen }\end{array}$ & $\begin{array}{l}\text { 1.4.2: } \\
\text { 1.4.3: }\end{array}$ & $\begin{array}{l}\text { Automatisierung } \\
\text { Kontrolle und Sicherstellung der Reinheit }\end{array}$ \\
\hline 1.1.7: & $\begin{array}{l}\text { Vergleichbarkeit der klinischen } \\
\text { Translation von Zelltherapieprodukten }\end{array}$ & $\begin{array}{l}\text { 1.4.4: } \\
\text { 1.4.5: }\end{array}$ & $\begin{array}{l}\text { Qualitätskontrollen } \\
\text { Weiterverarbeitung der Zellen }\end{array}$ \\
\hline 1.1.8: & Europaweites Register für hiPS-Zellen & 1.4.6: & Differenzierung zu Keimzellen \\
\hline 1.2 & Generierung von hiPS-Zellen & 1.5 & Kryokokonservierung \\
\hline 1.2.1: & Auswahlprozess für allogene Therapien & 1.5.1: & Verfahren \\
\hline 1.2.2: & Auswahlprozess für autologe Therapien & 1.5.2: & Einhaltung der Kühlkette \\
\hline 1.2.3: & $\begin{array}{l}\text { Gewinnung der somatischen } \\
\text { Ausgangszellen }\end{array}$ & $\begin{array}{l}\text { 1.5.3: } \\
1.5 .4:\end{array}$ & $\begin{array}{l}\text { Automatisierung } \\
\text { Auftauen der Proben }\end{array}$ \\
\hline 1.2.4: & $\begin{array}{l}\text { Charakterisierung für spätere } \\
\text { Qualitätskontrollen }\end{array}$ & & \\
\hline 1.2.5: & Reprogrammierungsmethoden & & \\
\hline 1.2.6: & Rückstellproben bei Genmodifikationen & & \\
\hline
\end{tabular}

\section{ETHISCHE ASPEKTE}

\begin{tabular}{|c|c|c|c|}
\hline 2.1 & Spende von Körpermaterialien & 2.4 & Risiko-/Belastung-Chancen-Bewertung \\
\hline $\begin{array}{l}2.1 .1: \\
2.1 .2: \\
2.1 .3: \\
2.1 .4: \\
2.1 .5: \\
2.1 .6: \\
2.1 .7: \\
2.1 .8:\end{array}$ & $\begin{array}{l}\text { Einwilligung } \\
\text { Umfang der Aufklärung } \\
\text { Verfahren zum Erhalt der Einwilligung } \\
\text { Nutzung digitaler Technologien bei der } \\
\text { dynamischen Einwilligung } \\
\text { Widerruf } \\
\text { Erzeugung von Keimzellen } \\
\text { Veröffentlichung des Gesamtgenoms } \\
\text { Aufwandsentschädigung }\end{array}$ & $\begin{array}{l}\text { 2.4.2: } \\
\text { 2.4.3: } \\
\text { 2.4.4: } \\
\text { 2.4.5: }\end{array}$ & $\begin{array}{l}\text { Verbesserung des individuellen } \\
\text { Gesundheitszustandes } \\
\text { Evaluierung der Risiken und Belastungen } \\
\text { Bewertung der Angemessenheit von } \\
\text { Risiken und Chancen } \\
\text { Nachsorge im Hinblick auf } \\
\text { Langzeitrisiken } \\
\text { Obduktion }\end{array}$ \\
\hline 2.2 & Klinische Forschung: Studienplanung & 2.5 & $\begin{array}{l}\text { Aufklärung und Einwilligung der } \\
\text { Studienteilnehmer }\end{array}$ \\
\hline $\begin{array}{l}\text { 2.2.1: } \\
\text { 2.2.2: } \\
\text { 2.2.3: } \\
\text { 2.2.4: }\end{array}$ & $\begin{array}{l}\text { Einbindung aller Stakeholder } \\
\text { Offenlegung von Interessenkonflikten } \\
\text { Ausschöpfung des Nutzenpotentials } \\
\text { Datenschutz }\end{array}$ & $\begin{array}{l}2.5 .1: \\
2.5 .2: \\
2.5 .3: \\
2.5 .4: \\
2.5 .5: \\
2.5 .6: \\
2.5 .7:\end{array}$ & $\begin{array}{l}\text { Einwilligung } \\
\text { Einwilligungsfähigkeit } \\
\text { Umfang der Aufklärung } \\
\text { Förderung der Forschungsmündigkeit } \\
\text { von Studienteilnehmern } \\
\text { Rekrutierung } \\
\text { Nebenbefunde } \\
\text { Obduktion }\end{array}$ \\
\hline 2.3 & Probandenauswahl & 2.6 & Gesellschaftliche Aspekte \\
\hline $\begin{array}{l}\text { 2.3.1: } \\
\text { 2.3.2: } \\
\text { 2.3.3: }\end{array}$ & $\begin{array}{l}\text { Rechtfertigung der Auswahlkriterien } \\
\text { Vulnerabilität } \\
\text { Aufwandsentschädigung }\end{array}$ & $\begin{array}{l}\text { 2.6.1: } \\
\text { 2.6.2: } \\
\text { 2.6.3: } \\
\text { 2.6.4: }\end{array}$ & $\begin{array}{l}\text { Fairer Interessenausgleich } \\
\text { Information der Öffentlichkeit } \\
\text { Partizipation von Stakeholdern } \\
\text { Durchführungsort von Studien }\end{array}$ \\
\hline
\end{tabular}

Abb. 1 Übersicht der ClinhiPS-Empfehlungen - Naturwissenschaftliche und ethische Aspekte 
3. RECHTLICHE ASPEKTE

\begin{tabular}{|c|c|c|c|}
\hline 3.1 & Allgemeines & 3.4 & Gentechnikrecht \\
\hline \multirow[t]{2}{*}{ 3.1.1: } & \multirow{2}{*}{$\begin{array}{l}\text { Frühzeitige Kontaktaufnahme mit den } \\
\text { zuständigen Behörden }\end{array}$} & \multirow{5}{*}{ 3.4.1: } & Organismen und Mikroorganismen \\
\hline & & & \multirow{4}{*}{$\begin{array}{l}\text { An den europäischen Gesetzgeber } \\
\text { Legaldefinitionen der biologischen Einheit, Vermeh- } \\
\text { rungs- und Übertragungsfähigkeit im Zusammen- } \\
\text { hang mit dem Organismusbegriff in Art. } 2 \mathrm{Nr} \text { I RL } \\
2001 / 18 / \mathrm{EG}\end{array}$} \\
\hline \multirow[t]{2}{*}{3.2} & Arzneimittelrecht & & \\
\hline & Arzneispezialitäten & & \\
\hline \multirow[t]{3}{*}{ 3.2.1: } & An den österreichischen Gesetzgeber & & \\
\hline & $\begin{array}{l}\text { Behebung der Unklarheiten bei der Auslegung } \\
\text { des ursprünglichen Arzneispezialitätenbegriffs } \\
\text { bzw. Adaptierung des Anwendungsbereichs des } \\
\text { Gewebesicherheitsgesetzes (GSG) }\end{array}$ & \multirow[t]{2}{*}{ 3.4.2: } & \multirow[t]{2}{*}{$\begin{array}{l}\text { An den europäischen Gesetzgeber } \\
\text { Legaldefinitionen der mikrobiologischen Einheit, } \\
\text { Vermehrungs- und Weitergabefähigkeit im Zusam- } \\
\text { menhang mit dem Mikroorganismusbegriff in } \\
\text { Art. } 2 \text { lit. a RL 2009/41/EG }\end{array}$} \\
\hline & Krankenhausausnahme & & \\
\hline 3.2.2: & $\begin{array}{l}\text { An den europäischen Gesetzgeber } \\
\text { Einheitliche Definitionen auf EU-Ebene }\end{array}$ & \multirow[t]{2}{*}{ 3.4.3: } & $\begin{array}{l}\text { An den deutschen Gesetzgeber } \\
\text { Legaldefinitionen der biologischen Einheit, Vermeh- }\end{array}$ \\
\hline \multirow[t]{3}{*}{ 3.2.3: } & $\begin{array}{l}\text { An den österreichischen Gesetzgeber } \\
\text { Klarstellung des Erfordernisses einer Betriebs- }\end{array}$ & & $\begin{array}{l}\text { rungs- und Übertragungsfähigkeit im Zusammen- } \\
\text { hang mit dem Organismusbegriff in } \$ 3 \mathrm{Nr}: 1 \text { des } \\
\text { Gentechnikgesetzes (GenTG) }\end{array}$ \\
\hline & $\begin{array}{l}\text { bewilligung nach \$ } 63 \text { AMG für ATMPs, die unter } \\
\text { die Krankenhausausnahme fallen }\end{array}$ & \multirow[t]{3}{*}{ 3.4.4: } & An den deutschen Gesetzgeber \\
\hline & Herstellungserlaubnis bzw. Betriebsbewilligung & & $\begin{array}{l}\text { Anpassung des Begriffs, , Mikroorganismen “ in } \\
\text { \& } 3 \text { Nr. la GenTG an den aktuellen Stand der }\end{array}$ \\
\hline \multirow[t]{2}{*}{ 3.2.4: } & An den österreichischen Gesetzgeber & & Technik \\
\hline & $\begin{array}{l}\text { Klarstellung des Erfordernisses einer Betriebs- } \\
\text { bewilligung nach \$ } 63 \text { AMG für ATMPs }\end{array}$ & \multirow[t]{2}{*}{ 3.4.5: } & $\begin{array}{l}\text { An den österreichischen Gesetzgeber } \\
\text { Neuformulierung des Begriffs ,.Organismen “ in }\end{array}$ \\
\hline \multirow[t]{3}{*}{ 3.2.5: } & An den österreichischen Gesetzgeber & & \& 4 Z. I GTG \\
\hline & $\begin{array}{l}\text { Klarstellung, ob Entnahmeeinrichtungen eine } \\
\text { Betriebsbewilligung } i . S . \text { d. } \$ 63 \text { AMG benötigen }\end{array}$ & \multirow[t]{2}{*}{ 3.4.6: } & $\begin{array}{l}\text { An den österreichischen Gesetzgeber } \\
\text { Neuformulierung des Begriffs ,,Mikroorganismen“" }\end{array}$ \\
\hline & Klinische Prüfung & & in $\$ 4 Z .2 G T G$ \\
\hline \multirow[t]{2}{*}{ 3.2.6: } & An den österreichischen Gesetzgeber & & Gentechnisch veränderte Organismen (GVO) \\
\hline & $\begin{array}{l}\text { Erweiterung des } \$ 40 \text { Abs. } 6 \text { AMG auf biotechnolo- } \\
\text { gisch bearbeitete Gewebeprodukte }\end{array}$ & \multirow[t]{3}{*}{ 3.4.7: } & $\begin{array}{l}\text { An den europäischen Gesetzgeber } \\
\text { Vereinheitlichung der Sprachfassungen des Anhangs }\end{array}$ \\
\hline \multirow[t]{2}{*}{ 3.2.7: } & \multirow{2}{*}{$\begin{array}{l}\text { An den österreichischen Gesetzgeber } \\
\text { Ersetzung des Begriffs ,Arzneimittel für Gen- } \\
\text { therapie " in } \$ 40 \text { Abs. } 6 \text { und Abs. } 8 \text { AMG durch den } \\
\text { arzneimittelrechtlichen Begriff, ,Gentherapeutikum “ }\end{array}$} & & $\begin{array}{l}\text { I A Teil I Nr. I RL 2001/18/EG und Anhangs I Teil A } \\
\text { Nr. I RL 2009/41/EG hinsichtlich des Begriffs der } \\
\text { Nukleinsäure- bzw. DNS-Rekombinationstechniken }\end{array}$ \\
\hline & & & Somatische Gentherapie \\
\hline \multirow{4}{*}{ 3.2.8: } & Pharmakovigilanz & \multirow[t]{3}{*}{ 3.4.8: } & An den österreichischen Gesetzgeber \\
\hline & $\begin{array}{l}\text { An den österreichischen Gesetzgeber } \\
\text { Klarstellung der Nichtanwendbarkeit des } \S 75 j\end{array}$ & & $\begin{array}{l}\text { Willensbildungsprozess in Bezug auf die Definition } \\
\text { der somatischen Gentherapie in } \$ 4 Z .24 \text { GTG }\end{array}$ \\
\hline & AMG auf zentral zugelassene Arzneimittel und & & Sonstiges \\
\hline & $\begin{array}{l}\text { Korrektur des Fehlverweises in } \$ 750 \text { AMG } \\
\text { HiPS-Zell-basierte Keimzellen }\end{array}$ & \multirow[t]{4}{*}{ 3.4.9: } & \multirow{4}{*}{$\begin{array}{l}\text { An den österreichischen Gesetzgeber } \\
\text { Korrektur des Verweises in } \$ 2 \text { Abs. } 3 \text { GTG }\end{array}$} \\
\hline 3.2.9: & $\begin{array}{l}\text { An den deutschen Gesetzgeber } \\
\text { Ausdrückliche Klarstellung der fehlenden } \\
\text { Arzneimitteleigenschaft für künstliche funktional } \\
\text { äquivalente Keimzellen }\end{array}$ & & \\
\hline 3.2.10: & $\begin{array}{l}\text { An den deutschen Gesetzgeber } \\
\text { Erfordernis einer Herstellungserlaubnis nach } \\
\S 13 \text { Abs. I S. } 1 \text { AMG }\end{array}$ & & \\
\hline 3.2.11: & $\begin{array}{l}\text { An den österreichischen Gesetzgeber } \\
\text { Ausdrückliche Klarstellung der fehlenden Arznei- } \\
\text { mitteleigenschaft von Keimzellen zu Fortpflan- } \\
\text { zungszwecken }\end{array}$ & & \\
\hline \multirow[t]{2}{*}{3.3} & Transplantations- und Geweberecht & 3.5 & $\begin{array}{l}\text { Stammzellen-, Embryonenschutz- und } \\
\text { Fortpflanzungsmedizinrecht }\end{array}$ \\
\hline & Begriffsbestimmungen & & HiPS-Zell-basierte Keimzellen \\
\hline \multirow[t]{2}{*}{ 3.3.1: } & $\begin{array}{l}\text { An den deutschen Gesetzgeber } \\
\text { Neuformulierung der Definition der Gewebe- } \\
\text { einrichtung in \& Ia Nr: } 8 \text { TPG }\end{array}$ & \multirow[t]{4}{*}{ 3.5.1: } & \multirow[t]{4}{*}{$\begin{array}{l}\text { An den deutschen Gesetzgeber } \\
\text { Schaffung eines deutschen Fortpflanzungs- } \\
\text { medizingesetzes }\end{array}$} \\
\hline & Anwendungsbereich des TPG und des GSG & & \\
\hline 3.3.2: & $\begin{array}{l}\text { An den deutschen Gesetzgeber } \\
\text { Schaffung eines deutschen Gewebesicherheits- } \\
\text { gesetzes }\end{array}$ & & \\
\hline 3.3.3: & $\begin{array}{l}\text { An den deutschen Gesetzgeber } \\
\text { Klarstellung des Anwendungsbereichs des TFG für } \\
\text { die Gewinnung von Blut und Blutbestandteilen zur } \\
\text { Herstellung von hiPS-Zellen und ihren Differenzie- } \\
\text { rungsderivaten sowie für die Anwendung von hiPS- } \\
\text { Zell-derivierten Blutprodukten }\end{array}$ & & \\
\hline
\end{tabular}

Abb. 2 Übersicht der ClinhiPS-Empfehlungen - Rechtliche Aspekte 
besondere Forscher, Kliniker, Unternehmer, Spender, Patienten, Ethikkommissionen und Regulierungsbehörden. Darüber hinaus richten sich die Empfehlungen an den deutschen, österreichischen und europäischen Gesetzgeber.

Generell gilt, dass alle notwendigen Arbeitsschritte in Übereinstimmung mit den anerkannten ethischen Prinzipien der Forschung und guten wissenschaftlichen Praxis sowie unter Beachtung der einschlägigen Gesetze und Vorschriften durchgeführt werden müssen.

Nicht Gegenstand der Empfehlungen sind haftungs- und sozialversicherungsrechtliche sowie patent- und lizenzrechtliche Aspekte. Zudem ist zu beachten, dass manche der hier gegebenen Empfehlungen sich im Laufe der Zeit ändern können, insbesondere mit Blick auf Sicherheitsaspekte.

\section{Naturwissenschaftliche Aspekte: Technisch-praktischer Umgang mit hiPS-Zellen und hiPS-Zell-basierten Produkten im Kontext der klinischen Anwendung am Menschen}

\subsection{Allgemeines}

\section{Empfehlung 1.1.1: Anwendung von Good Manufacturing Practice}

Alle notwendigen Arbeitsschritte, die im Folgenden aufgeführt werden, sind in Übereinstimmung mit Good Manufacturing Practice (GMP-konform) durchzuführen und sind über ein entsprechendes Qualitätsmanagementsystem zu kontrollieren und zu dokumentieren.

\section{Empfehlung 1.1.2: GMP-konforme Herstellung von Medien und Reagenzien} Alle verwendeten Medien und Reagenzien sind GMP-konform herzustellen, wobei vorzugsweise chemisch definierte und xeno-freie Bestandteile verwendet werden sollen.

\section{Empfehlung 1.1.3: Datenintegration}

Alle anfallenden Informationen und Daten während des Prozesses von der somatischen Zelle bis hin zum Therapieprodukt sind lückenlos in ein Datenmanagementsystem zu integrieren. Im gesamten Prozess einschließlich der Lagerung sind Verwechselungen durch die Verwendung von eindeutigen Identifikationskennungen (z. B. über Barcodes) und durch deren Überprüfung bei allen Prozessschritten auszuschließen.

\section{Empfehlung 1.1.4: Identifikation von Langzeitrisiken}

Es ist für Langzeit-Untersuchungen (Follow-Ups) Vorsorge zu treffen, sodass auch Langzeiteffekte durch Vergleich mit einer Rückstellprobe untersucht werden können. Daher sollen Rückstellproben der somatischen Ausgangszellen, der generierten hiPS-Zellen sowie eines gegebenenfalls kryokonservierbaren Therapieprodukts für retrospektive Analysen hergestellt und ausreichend lange gelagert werden.

\section{Empfehlung 1.1.5: Standardisierte Qualitätskontrollen}

Es sind - jeweils angepasst an den wissenschaftlichen Erkenntnisstand - standardisierte Qualitätskontrollen durchzuführen, die unter anderem Aussagen zur Toxizität, Tumorigenität, genetischen Stabilität sowie zur Funktionalität erlauben. 
Empfehlung 1.1.6: Implementierung zellbiologischer Sicherheitsmechanismen Um potenziellen Gefahren durch Fehlfunktionen des hiPS-Zell-Produkts im Körper (z. B. Tumorentwicklung, Fehlverteilung der Zellen) vorzubeugen, sollte überprüft werden, ob für die geplante Anwendung ein zellbiologischer Sicherheitsmechanismus sinnvoll ist, beispielsweise durch eine Immobilisierung der Zellen (z. B. Verkapselung) oder durch den Einsatz eines Suizid-Gens (z. B. Herpes Simplex Virus Thymidinkinase-Gen).

\section{Empfehlung 1.1.7: Vergleichbarkeit der klinischen Translation von Zelltherapieprodukten}

Es soll bereits bei der Planung einer klinischen Translation eine möglichst große Vergleichbarkeit mit Zelltherapieprodukten angestrebt werden, die in registrierten Studien (z. B. International Clinical Trials Registry Platform) verwendet werden. Dazu sollen - soweit wissenschaftlich sinnvoll - möglichst viele Prozesse entsprechend den veröffentlichten Studien standardisiert werden.

\section{Empfehlung 1.1.8: Europaweites Register für hiPS-Zellen}

Es sollte ein europaweites Register der Herstellung und Verwendung von GMPkonformen hiPS-Zellen eingerichtet werden, um die Qualität und Sicherheit durch Transparenz und Vergleichbarkeit zu verbessern. In diesem sollen Daten zu den hiPS-Zellen (z. B. Herstellung, Kultivierung, Qualität), den daraus abgeleiteten noch nicht zugelassenen Zelltherapieprodukten (z. B. Zelltyp, Differenzierung, Qualität) sowie zu Studien zur klinischen Anwendung am Menschen registriert werden.

\subsection{Generierung von hiPS-Zellen}

\section{Empfehlung 1.2.1: Auswahlprozess für allogene Therapien}

Im Fall allogener Therapieansätze sollen Spender und/oder ihre somatischen Ausgangszellen anwendungsbezogen auf Basis des aktuellen wissenschaftlichen Erkenntnisstandes untersucht und ausgewählt werden (z. B. Ermittlung des infektiösen Status, genomische Charakterisierung, HLA-Typisierung, Geschlecht, Alter).

\section{Empfehlung 1.2.2: Auswahlprozess für autologe Therapien}

Im Fall autologer Therapieansätze sollen die Patienten und/oder ihre somatischen Ausgangszellen anwendungsbezogen auf Basis des aktuellen wissenschaftlichen Erkenntnisstandes untersucht werden (z. B. Ermittlung des infektiösen Status, genomische Charakterisierung), um eine sachgerechte Therapieentscheidung zu treffen.

\section{Empfehlung 1.2.3: Gewinnung der somatischen Ausgangszellen}

Die Gewinnung der somatischen Ausgangszellen soll möglichst wenig invasiv sein. Zum Beispiel ist die Entnahme einer geringen Menge Blutes einer Hautbiopsie vorzuziehen, sofern beide Verfahren im Hinblick auf Gewinnung und Verwendung medizinisch und naturwissenschaftlich gleichwertig sind.

\section{Empfehlung 1.2.4: Charakterisierung für spätere Qualitätskontrollen}

Für spätere vergleichende Qualitätskontrollen (z. B. Identitätsfeststellung von Zwischen- und Endprodukten) ist eine ausreichende genomische Charakterisierung des somatischen Ausgangsmaterials vorzunehmen. 


\section{Empfehlung 1.2.5: Reprogrammierungsmethoden}

Die Reprogrammierungsmethode soll auf Basis des aktuellen wissenschaftlichen Erkenntnisstandes hinsichtlich der Sicherheit der generierten hiPS-Zellen für die Transplantation ausgewählt und durch entsprechende Qualitätskontrollen geprüft werden (z. B. Nachweis der Vektorfreiheit). Nach heutigem Stand sind DNA-freie, nicht-integrierende Reprogrammierungsmethoden (z. B. mRNA, Proteine) zu bevorzugen.

\section{Empfehlung 1.2.6: Rückstellproben bei Genmodifikationen}

Im Fall von Genmodifikationen (z. B. durch CRISPR/Cas9) sollen Rückstellproben auch der nicht genetisch veränderten Zellen angelegt werden.

\subsection{Expansion von hiPS-Zellen}

\section{Empfehlung 1.3.1: Auswahl des Expansionssystems}

Nach einer Bedarfsanalyse soll ein geeignetes Expansionssystem ausgewählt werden (z. B. Suspensionsbioreaktoren), um die benötigte Zellzahl bei minimaler genetischer Veränderung zu erreichen (z. B. durch Eliminierung von Selektionseffekten, möglichst kurze Zeit in Kultur).

\section{Empfehlung 1.3.2: Automatisierung}

Nach Möglichkeit sollen (teil-)automatisierte Systeme (z. B. Bioreaktoren, Zellkulturroboter) verwendet werden, um menschliche Einflüsse und Fehler zu reduzieren und die Effizienz zu erhöhen.

\section{Empfehlung 1.3.3: Qualitätskontrollen}

Für die Expansion sollen ausschließlich gemäß Abschn. 1.2 (Empfehlungen 1.2.11.2.6) generierte hiPS-Zellen verwendet werden. Die Sicherheit der Zellen für die Transplantation darf durch die Expansion nicht beeinträchtigt werden. Daher ist nach erfolgter Expansion auf Basis des aktuellen wissenschaftlichen Kenntnisstandes zu prüfen, ob die Kontaminationsfreiheit, Erhaltung der Pluripotenz, genetische und phänotypische Stabilität sowie Identität mit dem Ausgangsmaterial gegeben sind.

\subsection{Differenzierung von hiPS-Zellen}

\section{Empfehlung 1.4.1: Differenzierungsfaktoren}

Bei gleichwertiger biologischer Funktion sollen vorzugsweise Differenzierungsfaktoren mit großer Stabilität und geringer Variabilität verwendet werden (z. B. Verwendung sog. small molecules).

\section{Empfehlung 1.4.2: Automatisierung}

Nach Möglichkeit sollen (teil-)automatisierte Systeme (z. B. Pipettierroboter, Bioreaktoren) verwendet werden, um menschliche Einflüsse und Fehler zu reduzieren und die Effizienz zu erhöhen. 


\section{Empfehlung 1.4.3: Kontrolle und Sicherstellung der Reinheit}

Nach erfolgreicher Differenzierung ist die Reinheit zu kontrollieren (z. B. mittels durchflusszytometrischer Analyse); gegebenenfalls ist eine Aufreinigung (z. B. durch Sortierung der gewünschten Zelltypen) durchzuführen.

\section{Empfehlung 1.4.4: Qualitätskontrollen}

Für die Differenzierung sollen ausschließlich gemäß Abschn. 1.3 (Empfehlungen 1.3.1-1.3.3) expandierte hiPS-Zellen verwendet werden. Die Funktionalität und Sicherheit des (späteren) Zelltherapieprodukts für die Transplantation ist auf Basis des aktuellen wissenschaftlichen Kenntnisstandes zu gewährleisten. Insbesondere sind neben Kontaminationsfreiheit, Funktionalität, Reinheit und Tumorigenität auch genetische, epigenetische und phänotypische Stabilität sowie die Identität zu prüfen.

\section{Empfehlung 1.4.5: Weiterverarbeitung der Zellen}

Soweit die differenzierten Zellen weiter prozessiert werden (z. B. zur Generierung von Gewebekonstrukten), müssen auf Basis des aktuellen wissenschaftlichen Kenntnisstandes angemessene Vorkehrungen zur Gewährleistung von Qualität und Sicherheit des Produkts getroffen werden.

\section{Empfehlung 1.4.6: Differenzierung zu Keimzellen}

Aufgrund des aktuellen wissenschaftlichen Kenntnisstandes aus dem murinen System sollte wegen der zu befürchtenden Defekte eine Differenzierung in menschliche Keimzellen (Ei- und Samenzellen) zu Fortpflanzungszwecken zurzeit nicht angestrebt werden, selbst wenn dies von Rechts wegen nicht untersagt ist.

\subsection{Kryokonservierung}

\section{Empfehlung 1.5.1: Verfahren}

Für das Banking von hiPS-Zellen und ihrer Derivate sollen ausreichende Zellmengen im Kristallisationsverfahren kryokonserviert werden, wobei zur exakten und dokumentierten Abkühlung vorzugsweise Einfrierautomaten zu verwenden sind. Für adhärente hiPS-Zell-Derivate sind kristallisationsfreie Vitrifikationsmethoden empfehlenswert.

\section{Empfehlung 1.5.2: Einhaltung der Kühlkette}

Um einen Funktionalitätsverlust der gefrorenen Proben zu vermeiden, ist vom Zeitpunkt der Kryokonservierung an die permanente Einhaltung der Kühlkette sicherzustellen (unterhalb der Glasübergangstemperatur der hiPS-Zellen und hiPS-ZellProdukte in der Gasphase des Stickstoffs, z. B. $-140^{\circ} \mathrm{C}$ ).

\section{Empfehlung 1.5.3: Automatisierung}

Nach Möglichkeit sollen (teil-)automatisierte Systeme, zum Beispiel automatisierte Ein-/Auslagerungssysteme, verwendet werden, um menschliche Einflüsse und Fehler zu reduzieren und die Effizienz zu erhöhen. 


\section{Empfehlung 1.5.4: Auftauen der Proben}

Der Auftauprozess soll so schonend wie möglich für die Proben durchgeführt werden (z. B. schnelles Auftauen und Erwärmen auf $37^{\circ} \mathrm{C}$ ohne Überhitzen, unverzügliches Auswaschen der Kryomedien).

\section{Ethische Aspekte}

\subsection{Spende von Körpermaterialien}

\section{Empfehlung 2.1.1: Einwilligung}

Eine Aufbewahrung, Aufbereitung und Nutzung von Körpermaterialien zur Generierung von hiPS-Zellen sollte nur mit Einwilligung des betroffenen Spenders erfolgen.

\section{Empfehlung 2.1.2: Umfang der Aufklärung}

Potentielle Spender sollten aufgeklärt werden über alle bekannten aktuellen und - soweit möglich - auch künftigen Forschungsziele, insbesondere ob diese der Grundlagenforschung, der diagnostischen oder therapeutischen Forschung zuzuordnen sind, über Art und Umfang des entnommenen Gewebes und seine Aufbereitung, die Risiken der Entnahme, verantwortliche Person bzw. Institution, Rekontaktierungsmöglichkeiten, Datenschutzaspekte, insbesondere die Pseudonymisierung der Daten, die Möglichkeit von Nebenbefunden und den Umgang mit ihnen, das Widerrufsrecht, den Umgang mit Daten und Materialien nach dem Tod des Spenders, die Weitergabe von Materialien und personenbezogenen Daten an Dritte sowie alle Eigentumsfragen einschließlich einer möglichen Kommerzialisierung von hiPS-Zell-basierten Produkten. Die Spender sollten insbesondere darüber aufgeklärt werden, dass sie am Gewinn aus einer Kommerzialisierung nicht beteiligt werden und dass sich für sie aus der Spende kein individueller Nutzen ergibt.

\section{Empfehlung 2.1.3: Verfahren zum Erhalt der Einwilligung}

Der Spender sollte (auf der Basis der Aufklärung, s. Empfehlung 2.1.2) die Möglichkeit haben, sich zwischen einer einmaligen und einer dynamischen Einwilligung zu entscheiden. Bei der einmaligen Einwilligung erteilt der Spender seine informierte Einwilligung nur einmal anlässlich der Entnahme oder Übereignung der Zellen. Dabei sollten Möglichkeiten einer Beschränkung der Einwilligung für spezifische Verwendungsweisen (z. B. hinsichtlich einer Erzeugung von Keimzellen, s. unten Empfehlung 2.1.6) angeboten werden.

Beim dynamischen Einwilligungsverfahren ist von vornherein vorgesehen, dass der Spender wiederholt kontaktiert wird oder selbst (z. B. nach Information über eine Homepage) den Kontakt herstellt, um zu gegebenen Zeitpunkten der Verwendung seiner Zellen und Daten für spezifische neue Forschungsvorhaben zuzustimmen oder diese abzulehnen. Ein solches interaktives Vorgehen, das die Möglichkeit zu wiederholter Kommunikation bietet, ist angesichts der rapiden Entwicklung der 
Stammzellforschung empfehlenswert. Eine Rekontaktierung kann etwa für den Fall vereinbart werden, dass Forschungsziele den ursprünglich avisierten Rahmen überschreiten oder Materialien bzw. Daten an weitere Institutionen weitergegeben werden sollen.

\section{Empfehlung 2.1.4: Nutzung digitaler Technologien bei der dynamischen Einwilligung}

Die Anforderungen an ein Rekontaktierungsverfahren sollten den Rahmen des Zumutbaren nicht überschreiten. Zwar liegt die Verantwortung für die angemessene Information der Spender bzw. für die angemessene Bereitstellung von Informationen zum Beispiel auf einer Homepage oder über eine App bei der verantwortlichen Institution; die Einleitung der darauf folgenden Schritte innerhalb einer angemessenen bzw. vereinbarten Zeitspanne (persönliche Kontaktaufnahme, Widerruf, Abruf der Information über Nebenbefunde etc.) kann dagegen beim Spender verbleiben. Es wird empfohlen, zur Kontaktherstellung digitale Technologien wie Apps zu nutzen bzw. zu entwickeln, die allen datenschutzrechtlichen Anforderungen entsprechen.

\section{Empfehlung 2.1.5: Widerruf}

Die Einwilligung zur Verwendung von gespendeten Biomaterialien kann vor der Herstellung von hiPS-Zellen jederzeit und ohne Angabe von Gründen widerrufen werden.

Für den Fall, dass bereits hiPS-Zellen aus den gespendeten Biomaterialien hergestellt wurden, kann die Möglichkeit des Spenders ausgeschlossen werden, die Vernichtung dieser hiPS-Zellen zu verlangen oder ihre (weitere) Verwendung für die medizinische Forschung zu untersagen. Der Spender ist über einen solchen Ausschluss aufzuklären. Die Möglichkeit des Widerrufs der Einwilligung in die Herstellung neuer hiPS-Zellen aus dem gespendeten Biomaterial (Ausgangsmaterial) bleibt davon unberührt.

Zum Nachweis der Zell-Linien-Identität und für Rückstellproben sollte Ausgangsmaterial erhalten werden. Die lagernde Biobank sollte die langfristige Aufbewahrung sicherstellen.

\section{Empfehlung 2.1.6: Erzeugung von Keimzellen}

Der Versuch der Erzeugung von Keimzellen aus dem gespendeten Material - soweit überhaupt rechtlich zulässig - bedarf einer ausdrücklichen Einwilligung des Spenders.

\section{Empfehlung 2.1.7: Veröffentlichung des Gesamtgenoms}

Die Veröffentlichung der Gesamtheit der Erbinformation (des Gesamtgenoms) des Spenders bedarf einer ausdrücklichen Einwilligung des Spenders.

\section{Empfehlung 2.1.8: Aufwandsentschädigung}

Sofern die Spende für den Spender mit einem besonderen Aufwand verbunden ist (z. B. gesonderte Anfahrt), sollte eine finanzielle Kompensation in Form einer Aufwandsentschädigung in Betracht gezogen werden. Diese darf keinen unangemessenen Anreiz für die Spende darstellen. 


\subsection{Klinische Forschung: Studienplanung}

\section{Empfehlung 2.2.1: Einbindung aller Stakeholder}

Um eine faire Durchführung der Studie zu ermöglichen, sollten alle beteiligten Stakeholder, insbesondere Patienten(vertreter), möglichst frühzeitig in den konzeptionellen Planungsprozess einbezogen werden. Dabei sollte insbesondere eine Bewertung von Risiken, Belastungen und Nutzen auch durch potenzielle Studienteilnehmer erfolgen.

\section{Empfehlung 2.2.2: Offenlegung von Interessenkonflikten}

Interessenkonflikte, die zu einer verzerrten Bewertung der Chancen und Risiken des Forschungsvorhabens führen können, sind gegenüber den potenziellen Studienteilnehmern offenzulegen.

\section{Empfehlung 2.2.3: Ausschöpfung des Nutzenpotenzials}

Jeder Studie sollte ein Konzept zugrunde liegen, das den transparenten Umgang mit positiven wie negativen Ergebnissen der Forschungsmaßnahme einschließlich ihrer möglichen klinischen Anwendung umfasst.

Die Studienrohdaten sollten unter Wahrung der Anonymität und berechtigter Belange geistigen Eigentums für die wissenschaftliche Zweitauswertung zur Verfügung gestellt werden.

\section{Empfehlung 2.2.4: Datenschutz}

Jeder Studie sollte ein Konzept zum Datenschutz zugrunde liegen, da bei allen Forschungsmaßnahmen und gegenüber allen Nutzern der Schutz personenbezogener Daten gewährleistet sein muss.

\subsection{Probandenauswahl}

\section{Empfehlung 2.3.1: Rechtfertigung der Auswahlkriterien}

Die Auswahl der Probandenpopulation sollte auf der Basis einer Risiko-/BelastungChancen-Bewertung (s. unten Abschn. 2.4) im Studienprotokoll eigens gerechtfertigt werden. Sie sollte nicht allein nach technisch-praktischen Kriterien (z. B. einfache Rekrutierung) erfolgen.

Es sollte insbesondere auch dargelegt werden, welche spezifischen Risiken und Belastungen einerseits sowie Chancen andererseits sich für die ausgewählte Population im Vergleich zu anderen möglichen Probandenpopulationen ergeben und ob und inwiefern für die spezifische Probandengruppe alternative Behandlungsformen zur Verfügung stehen.

\section{Empfehlung 2.3.2: Vulnerabilität}

Es sollte dargelegt werden, ob und in welcher Hinsicht die ausgewählte Population vulnerabel ist (z. B. aufgrund mangelnder Einwilligungsfähigkeit, hohen Alters, Alternativlosigkeit bei unheilbarer Erkrankung, Komorbidität). Entsprechend ist dar- 
zulegen, welche spezifischen Maßnahmen des Schutzes und Empowerments vorgesehen sind.

\section{Empfehlung 2.3.3: Aufwandsentschädigung}

In Phase I/II sollte eine finanzielle Kompensation in Form einer Aufwandsentschädigung der Studienteilnehmer in Betracht gezogen werden. Diese darf keinen unangemessenen Anreiz für eine Studienteilnahme darstellen.

\subsection{Risiko-/Belastung-Chancen-Bewertung}

\section{Empfehlung 2.4.1: Verbesserung des individuellen Gesundheitszustandes}

Studien sollen grundsätzlich so angelegt sein, dass sie zur Verbesserung des Gesundheitszustands der teilnehmenden Patienten beitragen können. Präklinische Studien müssen verlässliche Anhaltspunkte dafür ergeben haben, dass eine klinische Anwendung die Aussicht auf Heilung einer Krankheit oder spürbare Verbesserung von Krankheitssymptomen verspricht. Dabei müssen sich auch Anhaltspunkte für die Überlegenheit des neuen Therapieverfahrens gegenüber alternativen Verfahren ergeben haben.

Ist ein gesundheitlicher Nutzen für den Patienten nicht oder nur mit geringer Wahrscheinlichkeit zu erwarten, muss zumindest ein substanzieller wissenschaftlicher Nutzen wahrscheinlich sein.

Ein fehlender individueller Nutzen darf nicht durch unangemessene finanzielle Anreize kompensiert werden.

\section{Empfehlung 2.4.2: Evaluierung der Risiken und Belastungen}

Mit der Studie einhergehende Risiken und Belastungen sind zu identifizieren und zu bewerten. Dabei sollten kurzfristige und langfristige Risiken gesondert bewertet werden. Die Belastungen des Versuchs (Anzahl der Folgeuntersuchungen, LangzeitFollow-up etc.) sollten eigens evaluiert werden.

Die Risiken und Belastungen für die Vergleichsgruppe sollten stets gesondert bewertet werden, auch und insbesondere dann, wenn es sich um gesunde Probanden handelt. Der Vergleich gegen Standardtherapie oder Placebo bzw. Scheininterventionen setzt klinische Equipoise (Gleichwertigkeit) voraus.

\section{Empfehlung 2.4.3: Bewertung der Angemessenheit von Risiken und Chancen}

Die mit einer Studie einhergehenden Risiken und Belastungen müssen in einem angemessenen Verhältnis zum erwarteten individuellen und gesellschaftlichen Nutzen stehen. Die Bewertung dessen, was als angemessenes Verhältnis zwischen Risiken und Chancen erachtet wird, hängt maßgeblich von unterschiedlichen Perspektiven und Interessen ab. Eine entsprechende Beteiligung der betreffenden Stakeholder, insbesondere von Vertretern der betroffenen Patienten/Probandenpopulation, ist daher unerlässlich. Die Bewertung des Risiko-Chancen-Verhältnisses soll unter Berücksichtigung einer maximalen Risiko- sowie einer minimalen Nutzenschwelle erfolgen. 


\section{Empfehlung 2.4.4: Nachsorge im Hinblick auf Langzeitrisiken}

Es muss in die Risikobewertung einbezogen werden, inwieweit eine im Hinblick auf die jeweilige Studie zeitlich angemessene Nachsorge für mit der Studie in Zusammenhang stehenden Langzeitrisiken erforderlich ist. Die Langzeitrisiken einschließlich der Nachsorgemaßnahmen müssen vom Versicherungsschutz der Studie mit umfasst sein.

\section{Empfehlung 2.4.5: Obduktion}

Wenn Anlass zu der Vermutung besteht, dass der Tod eines Patienten auf die Studie zurückzuführen ist, wird die Obduktion des Patienten empfohlen, soweit diese nicht ohnehin - wie unter bestimmten Voraussetzungen in Österreich - von Rechts wegen gefordert ist. Das Ergebnis sollte Bestandteil der wissenschaftlichen Auswertung und Veröffentlichung der Studienergebnisse sein.

\subsection{Aufklärung und Einwilligung der Studienteilnehmer}

\section{Empfehlung 2.5.1: Einwilligung}

Die Studienteilnahme bedarf einer Einwilligung des Studienteilnehmers nach einer umfassenden Aufklärung über alle studienspezifischen Aspekte (s. unten Empfehlung 2.5.3).

\section{Empfehlung 2.5.2: Einwilligungsfähigkeit}

Es sollte im Studienprotokoll dargelegt werden, wie die Einwilligungsfähigkeit der Studienteilnehmer festgestellt wird. Studien an Nichteinwilligungsfähigen sollten nach Möglichkeit subsidiär erfolgen, das heißt erst wenn Studien an Einwilligungsfähigen eine hinreichende Wahrscheinlichkeit für ein angemessenes Risiko-/Belastung-Chance-Verhältnis ergeben haben.

Bei Studienteilnehmern, die nicht einwilligungsfähig sind, sollten insbesondere die Belastungen durch die Studie aus Sicht der Betroffenen sorgfältig erhoben werden. Studienteilnehmer, die zur Bildung einer eigenen Meinung in der Lage sind (z. B. kleinere Kinder), sollten Gelegenheit erhalten, ihre Bedürfnisse in die Ausgestaltung der studienbedingten Maßnahmen einzubringen.

\section{Empfehlung 2.5.3: Umfang der Aufklärung}

Studienteilnehmer sind umfassend aufzuklären, insbesondere darüber, welche kurzund langfristigen Risiken mit der Studienteilnahme verbunden sind und wie sie gegen den erhofften Nutzen abgewogen werden. Es sollte auch darüber aufgeklärt werden, welche Belastungen sich durch die Studienteilnahme ergeben. Hierzu gehört auch eine ausführliche Darlegung der Belastungen durch das long-term follow-up.

Dabei sollte auch darauf verwiesen werden, mit welcher Wahrscheinlichkeit die Studienteilnehmer einen Nutzen von der Teilnahme an der Studie erwarten können. Hierbei sollten Maßnahmen ergriffen werden, die einer fehlerhaften Einschätzung einer rein wissenschaftlichen Forschung als therapeutischer Forschung (,therapeutic misconception“) oder einer Fehleinschätzung des individuellen therapeutischen Nutzens (,therapeutic misestimation“) gezielt entgegenwirken. 


\section{Empfehlung 2.5.4: Förderung der Forschungsmündigkeit von Studienteilnehmern}

Die Fähigkeit von Studienteilnehmern, grundlegende Aspekte eines Forschungsvorhabens, insbesondere solche, die relevante Auswirkungen auf ihr eigenes Leben haben, beurteilen zu können (Forschungsmündigkeit), sollte gefördert werden. Aufklärungsmaterialien sollten in Zusammenarbeit mit Patientenvertretern bzw. Angehörigenvertretern erstellt werden, um die Bedürfnisse von Studienteilnehmer besser zu berücksichtigen. Es sollte eine Kurzfassung der wichtigsten Aspekte der Studie in laienverständlicher Sprache beigefügt werden. Dazu wird eine frühzeitige Einbindung von einschlägigen Patientengruppen empfohlen.

\section{Empfehlung 2.5.5: Rekrutierung}

Die Rekrutierung von Studienteilnehmern sollte möglichst in Zusammenarbeit mit Patienten- oder anderen Selbsthilfeorganisationen erfolgen. Der Rekrutierungsprozess sollte mindestens aus je einem Informationsgespräch mit einem geschulten Patienten- bzw. Angehörigenvertreter und einem Leiter der Studie bestehen.

\section{Empfehlung 2.5.6: Nebenbefunde}

Über die Möglichkeit und Konsequenzen von Nebenbefunden ist rechtzeitig zu informieren. Mit dem Studienteilnehmer ist zu vereinbaren, ob über Nebenbefunde aufgeklärt werden soll oder nicht.

\section{Empfehlung 2.5.7: Obduktion}

Wenn Anlass zu der Befürchtung besteht, dass die Therapie zum Tod eines Patienten führen könnte, sollte die Studienteilnahme möglichst an die Bereitschaft zu einer Obduktion im Todesfall gekoppelt werden, für die eine ausdrückliche Aufklärung und Einwilligung eingeholt werden sollte. Dies gilt nicht, wenn die Obduktion - wie unter bestimmten Voraussetzungen in Österreich - von Rechts wegen ohnehin zulässig ist.

\subsection{Gesellschaftliche Aspekte}

\section{Empfehlung 2.6.1: Fairer Interessenausgleich}

Stammzellforschung beruht in der Regel auf der Kooperation zwischen einer Vielzahl sehr unterschiedlicher Stakeholder, die unterschiedliche Interessen repräsentieren, insbesondere von kommerziellen und nicht kommerziellen Akteuren. Die Ziele der Forschung sollten die Interessen aller Stakeholder angemessen widerspiegeln; insbesondere sollte diese Art der klinischen Stammzellforschung einen direkten Nutzen für die gesundheitliche Versorgung der Bevölkerung versprechen. Forschungsvorhaben, die einen primär kommerziellen Nutzen anstreben, sollten eine Kompensation für öffentliche Institutionen vorsehen, um die solidarisch erbrachten Beiträge von Spendern und Patienten anzuerkennen.

\section{Empfehlung 2.6.2: Information der Öffentlichkeit}

Die Öffentlichkeit sollte ausreichend Zugang zu weiterführenden Informationen über die Stammzellforschung erhalten, die sie zu einem kompetenten Umgang mit den 
verschiedenen Optionen der Forschung befähigen. Solche weiterführenden Informationen sollten von einer unabhängigen Einrichtung bereitgestellt werden. Sie sollten sowohl medizinisch-technische als auch ethische, rechtliche und soziale Aspekte angemessen berücksichtigen. Wir empfehlen beispielsweise, dass öffentliche Stellen, die über Organ- und Gewebespende informieren, die Spende von Zellen/Gewebe für die hiPS-Zell-Forschung in ihren Informationsmaterialien berücksichtigen.

Es wird empfohlen, Studienergebnisse auch in einer laienverständlichen Sprache zu veröffentlichen, um die Verbreitung von Wissen über Stammzellforschung in der Öffentlichkeit zu verbessern.

\section{Empfehlung 2.6.3: Partizipation von Stakeholdern}

Forschungsförderern wird empfohlen, Maßnahmen des Stakeholder Involvements gezielt zu fördern, um die Partizipation von Betroffenengruppen in unterschiedlichen Phasen der klinischen Translation zu ermöglichen.

Es wird angeregt, über besonders sensible Aspekte der Stammzellforschung, etwa die Erzeugung von menschlichen Keimzellen aus Stammzellen, eine breite gesellschaftliche Debatte zu initiieren, um den politischen Entscheidungsprozess besser zu fundieren.

\section{Empfehlung 2.6.4: Durchführungsort von Studien}

Risikobehaftete klinische Stammzellforschung sollte möglichst in jenen Ländern erfolgen, deren Patienten voraussichtlich von den derart entwickelten Therapien profitieren werden.

\section{Rechtliche Aspekte}

\subsection{Allgemeines}

\section{Empfehlung 3.1.1: Frühzeitige Kontaktaufnahme mit den zuständigen Behörden}

Es wird empfohlen, bei der Entwicklung und Anwendung von hiPS-Zell-basierten Produkten frühzeitig mit den zuständigen Behörden Kontakt aufzunehmen, insbesondere im Hinblick darauf, ob eine Zulassung, Genehmigung, Erlaubnis, Bewilligung und/oder Anmeldung erforderlich ist.

\subsection{Arzneimittelrecht}

\section{Arzneispezialitäten}

An den österreichischen Gesetzgeber

Empfehlung 3.2.1: Behebung der Unklarheiten bei der Auslegung des ursprünglichen Arzneispezialitätenbegriffs bzw. Adaptierung des Anwendungsbereichs des Gewebesicherheitsgesetzes (GSG) 
$\S 1$ Abs. 5 Arzneimittelgesetz (AMG) enthält einen Arzneispezialitätenbegriff, der historisch in zwei Schichten gewachsen ist (einen „ursprünglichen“ und einen „erweiterten“ Arzneispezialitätenbegriff). Das AMG und andere Rechtsvorschriften differenzieren hinsichtlich der Rechtsfolgen zwischen diesen beiden Begriffen. Insbesondere hängt davon die Reichweite des Anwendungsbereichs des GSG nach § 1 Abs. 1 GSG ab. Für Arzneispezialitäten i. S. d. ursprünglichen Definition sieht $§ 1$ Abs. 1 S. 2 GSG eine Teilausnahme vom Anwendungsbereich vor. Ausschließlich industriell oder gewerblich hergestellte Arzneispezialitäten (erweiterter Arzneispezialitätenbegriff) unterliegen hingegen uneingeschränkt dem Anwendungsbereich des GSG. Nach derzeitiger Rechtslage sind nicht alle hiPS-Zell-basierten Produkte als Arzneispezialitäten i. S. d. ursprünglichen Arzneispezialitätenbegriffs einzustufen. Dies trifft (mangels Herstellung im Voraus) vor allem auf autologe Produkte zu. Für diese gelten das AMG und das GSG (zur Gänze) grundsätzlich kumulativ; das AMG normiert aber zum Teil die Spezialität der geweberechtlichen Regelungen. Dieser Vorrang der geweberechtlichen Bestimmungen steht hinsichtlich Arzneimittel für neuartige Therapien (ATMPs) bei der arzneimittelrechtlichen Betriebsbewilligung im Widerspruch zu den Anforderungen der Verordnung (VO) (EG) 1394/2007 (ATMP-VO).

Der Gesetzgeber sollte daher insbesondere die Reichweite des Anwendungsbereichs des GSG im Hinblick auf das Verhältnis zum AMG überdenken und allenfalls klarstellen. Falls nicht gewünscht ist, dass das GSG auf (ausschließlich) industriell oder gewerblich hergestellte ATMPs voll anwendbar ist, sollte die Teilausnahme des $\S 1$ Abs. 1 S. 2 GSG entsprechend adaptiert werden. Dies stünde auch im Einklang mit den unionsrechtlichen Vorgaben, nach denen die geweberechtlichen Vorschriften nur für die Gewinnung, die arzneimittelrechtlichen Anforderungen der ATMPVO für alle weiteren Aspekte gelten sollen (vgl. ErwG 14 ATMP-VO).

\section{Krankenhausausnahme}

Gemäß Art. 3 Nr. 7 Richtlinie (RL) 2001/83/EG (i. V. m. Art. 28 Nr. 2 ATMP-VO) sind ATMPs, ,die nicht routinemäßig nach spezifischen Qualitätsnormen hergestellt und in einem Krankenhaus in demselben Mitgliedstaat unter der ausschließlichen fachlichen Verantwortung eines Arztes auf individuelle ärztliche Verschreibung eines eigens für einen einzelnen Patienten angefertigten Arzneimittels verwendet werden“, vom Anwendungsbereich der ATMP-VO und der RL 2001/83/EG ausgenommen (sog. Krankenhausausnahme). Sie unterliegen daher insbesondere nicht der zentralen Zulassung durch die europäische Kommission.

\section{An den europäischen Gesetzgeber}

\section{Empfehlung 3.2.2: Einheitliche Definitionen auf EU-Ebene}

Dem europäischen Gesetzgeber wird empfohlen, den Begriff „,nicht routinemäBig (...) hergestellt" in Art. 3 Nr. 7 RL 2001/83/EG (i. V. m. Art. 28 Nr. 2 ATMP-VO) zu definieren. Darüber hinaus sollte klargestellt werden, ob dem Begriff „Krankenhaus“" in Art. 3 Nr. 7 RL 2001/83/EG (i. V. m. Art. 28 Nr. 2 ATMP-VO) ein enges Verständnis zu Grunde zu legen ist oder ob auch die ambulante Anwendung umfasst sein soll. 


\section{An den österreichischen Gesetzgeber}

Empfehlung 3.2.3: Klarstellung des Erfordernisses einer Betriebsbewilligung nach \$ 63 AMG für ATMPs, die unter die Krankenhausausnahme fallen

Art. 3 Nr. 7 RL 2001/83/EG (i. V. m. Art. 28 Nr. 2 ATMP-VO) verlangt für die Herstellung von ATMPs, die unter die Krankenhausausnahme fallen, eine arzneimittelrechtliche Genehmigung. Problematisch ist, dass $§ 62$ Abs. 2 Z. 6 AMG Gewebebanken, die dem vollen Anwendungsbereich des GSG unterliegen (s. Empfehlung 3.2.1), vom Erfordernis einer arzneimittelrechtlichen Betriebsbewilligung gemäß § 63 AMG ausnimmt. Diese Ausnahme erfasst auch Gewebebanken, die unter die Krankenhausausnahme fallende ATMPs herstellen. Eine Befreiung von der Betriebsbewilligungspflicht steht im Widerspruch zu den unionsrechtlichen Anforderungen. Dem österreichischen Gesetzgeber wird daher empfohlen, die Ausnahme von der Betriebsbewilligung in $\S 62 \mathrm{Abs} .2 \mathrm{Z}$. $6 \mathrm{AMG}$ an das Unionsrecht anzupassen, beispielsweise in Form einer generellen Gegenausnahme. Bis dahin sollte die Vollzugsbehörde, das Bundesamt für Sicherheit im Gesundheitswesen (BASG), die volle Wirksamkeit des Unionsrechts gewährleisten und $\S 62$ Abs. 2 Z. 6 AMG im Falle der Herstellung von unter die Krankenhausausnahme fallenden ATMPs unangewendet lassen. Den Herstellern sämtlicher hiPS-Zell-basierter Produkte wird in jedem Fall die Einholung einer Betriebsbewilligung nach § 63 AMG nahegelegt.

\section{Herstellungserlaubnis bzw. Betriebsbewilligung}

\section{An den österreichischen Gesetzgeber}

Empfehlung 3.2.4: Klarstellung des Erfordernisses einer Betriebsbewilligung nach § 63 AMG für ATMPs

Problematisch ist die Ausnahmebestimmung des $§ 62$ Abs. 2 Z. 6 AMG auch im Hinblick auf nicht unter die Krankenhausausnahme fallende, ausschließlich industriell oder gewerblich hergestellte ATMPs (das heißt ATMPs, die nicht unter den ursprünglichen Arzneispezialitätenbegriff gemäß $§ 1$ Abs. 5 AMG fallen). Für diese ergibt sich das Erfordernis einer Herstellungserlaubnis aus Art. 19 Abs. 1 VO (EG) 726/2004 i. V. m. Art. 40 RL 2001/83/EG. Daher wird dem österreichischen Gesetzgeber empfohlen, die Ausnahme von der Betriebsbewilligung in $\S 62$ Abs. 2 Z. 6 AMG an das Unionsrecht anzupassen, beispielsweise in Form einer generellen Gegenausnahme für solche ATMPs (s. dazu auch Empfehlung 3.2.3). Bis dahin sollte die Vollzugsbehörde, das BASG, §62 Abs. 2 Z. 6 AMG im Einklang mit den unionsrechtlichen Anforderungen handhaben und vom Erfordernis einer Betriebsbewilligung nach $\S 63$ AMG ausgehen. Den Herstellern sämtlicher hiPS-Zell-basierter Produkte wird in jedem Fall die Einholung einer Betriebsbewilligung nach $\S 63$ AMG nahegelegt.

Empfehlung 3.2.5: Klarstellung, ob Entnahmeeinrichtungen eine Betriebsbewilligung i. S. d. \$ 63 AMG benötigen

Die Ausnahmebestimmung des $§ 62$ Abs. 2 Z. 6 AMG erfasst nur Gewebebanken, nicht aber Entnahmeeinrichtungen i. S. d. GSG. Diese unterliegen nach strenger Wortlautinterpretation der Betriebsbewilligungspflicht nach $\S 63$ AMG. Die besseren Gründe sprechen aber dafür, auch Entnahmeeinrichtungen von der arznei- 
mittelrechtlichen Betriebsbewilligungspflicht auszunehmen. Dem österreichischen Gesetzgeber wird empfohlen, die Rechtslage hinsichtlich Entnahmeeinrichtungen zu klären und diese gegebenenfalls ausdrücklich vom Erfordernis einer arzneimittelrechtlichen Betriebsbewilligung nach § 63 AMG auszunehmen. Dies könnte durch eine Erweiterung des $\S 62$ Abs. 2 AMG erfolgen.

\section{Klinische Prüfung}

\section{An den österreichischen Gesetzgeber}

Empfehlung 3.2.6: Erweiterung des $\$ 40$ Abs. 6 AMG auf biotechnologisch bearbeitete Gewebeprodukte

Nach § 40 Abs. 6 AMG bedürfen klinische Prüfungen ,im Zusammenhang mit Arzneimitteln für Gentherapie und somatische Zelltherapie“ einer Genehmigung des BASG. Im Hinblick auf die unionsrechtlichen Anforderungen wird dem österreichischen Gesetzgeber empfohlen, ein Genehmigungserfordernis auch für klinische Prüfungen mit biotechnologisch bearbeiteten Gewebeprodukten zu verankern. Dies könnte durch eine Erweiterung des § 40 Abs. 6 AMG im Zuge der ohnehin notwendigen Anpassung an die VO (EU) 536/2014 über klinische Prüfungen mit Humanarzneimitteln erfolgen. Bis dahin ergibt sich die Genehmigungspflicht unmittelbar aus Art. 4 Abs. 1 ATMP-VO i. V. m. Art. 9 Abs. 6 RL 2001/20/EG. Hersteller bzw. Anwender müssen daher stets eine Genehmigung für klinische Prüfungen mit biotechnologisch bearbeiteten Gewebeprodukten einholen.

Empfehlung 3.2.7: Ersetzung des Begriffs „Arzneimittel für Gentherapie“ in $\S 40$ Abs. 6 und Abs. 8 AMG durch den arzneimittelrechtlichen Begriff „Gentherapeutikum"

Klinische Prüfungen mit „Arzneimitteln für Gentherapie“ unterliegen gemäß $\S 40$ Abs. 8 AMG auch den Anforderungen der $\S \S 74-79$ Gentechnikgesetz (GTG). Sie bedürfen insbesondere einer zusätzlichen Bewilligung des Bundesministers für Gesundheit. Vor dem Hintergrund des unterschiedlichen Verständnisses der „Gentherapie“ nach Arzneimittelrecht und nach Gentechnikrecht (s. auch Empfehlung 3.4.8) wird dem österreichischen Gesetzgeber empfohlen, den Begriff „Arzneimittel für Gentherapie“ in $\S 40$ Abs. 6 und 8 AMG durch den arzneimittelrechtlichen Begriff „Gentherapeutikum“ zu ersetzen.

\section{Pharmakovigilanz}

\section{An den österreichischen Gesetzgeber}

Empfehlung 3.2.8: Klarstellung der Nichtanwendbarkeit des § 75j AMG auf zentral zugelassene Arzneimittel und Korrektur des Fehlverweises in \$ 75o AMG

Im Rahmen der Pharmakovigilanz sieht das AMG Meldepflichten für Angehörige der Gesundheitsberufe ( $\$ 75 \mathrm{~g} \mathrm{AMG})$ und Zulassungsinhaber ( $\$ 75 \mathrm{j} A M G)$ vor. $\S 75 \mathrm{j}$ AMG differenziert seinem Wortlaut nach nicht zwischen zentral und auf sonstige Weise zugelassenen Arzneispezialitäten; er enthält insbesondere keine explizite Ausnahmeregelung für zentral zugelassene Arzneispezialitäten. Historische und unionsrechtskonforme Interpretation lassen aber den Schluss zu, dass sich $\S 75 \mathrm{j}$ AMG nur auf nationale Meldepflichten bezieht. In Bezug auf zentral zugelassene Arzneispezialitäten ergeben sich (inhaltlich identische) Meldepflichten des Zulassungsinhabers unmittelbar aus Art. 28 Abs. 1 VO (EG) 726/2004 i. V. m. Art. 107 
RL 2001/83/EG. Im Sinne der Rechtsklarheit sollte der Gesetzgeber die Nichtanwendbarkeit des $\S 75$ j AMG (und einen entsprechenden Verweis auf die VO [EG] 726/2004) für zentral zugelassene Arzneispezialitäten explizit verankern. Damit wäre gleichzeitig klargestellt, dass eine Verdrängung der arzneimittel- durch die geweberechtlichen Meldepflichten gemäß § 75o AMG nicht in Betracht kommt.

Bei dieser Gelegenheit sollte der Gesetzgeber auch den Fehlverweis (auf die frühere Fassung der arzneimittelrechtlichen Meldepflichten gemäß $\S 75 \mathrm{a}$ bis $75 \mathrm{c}$ $\mathrm{AMG}$ ) in $\S 750$ AMG berichtigen.

\section{HiPS-Zell-basierte Keimzellen}

\section{An den deutschen Gesetzgeber}

Empfehlung 3.2.9: Ausdrückliche Klarstellung der fehlenden Arzneimitteleigenschaft für künstliche funktional äquivalente Keimzellen

$\S 4$ Abs. 30 S. 2 AMG legt fest, dass Keimzellen (d. h. menschliche Samen- und Eizellen) keine Arzneimittel und auch keine Gewebezubereitungen i. S. d. AMG sind. Keimzellen sind allerdings „Gewebe“ i. S. d. § 1a Nr. 4 des Transplantationsgesetzes (TPG).

Dem deutschen Gesetzgeber wird empfohlen, ausdrücklich klarzustellen, dass sich § 4 Abs. 30 S. 2 AMG auch auf in vitro hergestellte menschliche Zellen bezieht, die funktional äquivalent zu natürlich entstandenen Keimzellen sind (nachfolgend: „,künstliche funktional äquivalente Keimzellen“) und zu Fortpflanzungszwecken bestimmt sind.

Empfehlung 3.2.10: Erfordernis einer Herstellungserlaubnis nach $\$ 13$ Abs. 1

\section{S. 1 AMG}

Falls der deutsche Gesetzgeber daran festhält, dass Keimzellen als „Gewebe“ i. S. d. § 1a Nr. 4 TPG in den Anwendungsbereich des AMG fallen, dann sollte er zur Gewährleistung hoher Qualitäts- und Sicherheitsstandards für die Herstellung von künstlichen funktional äquivalenten hiPS-Zell-basierten Keimzellen zu Fortpflanzungszwecken eine Herstellungserlaubnis nach $\S 13$ Abs. 1 S. 1 AMG fordern. Nur durch eine Erlaubnis nach $\S 13$ Abs. 1 S. 1 AMG ist die gewerbs- oder berufsmäßige Herstellung von künstlichen funktional äquivalenten hiPS-Zell-basierten Keimzellen zu Fortpflanzungszwecken nach dem GMP-Standard gesichert. Zu weiteren Regelungen s. Empfehlung 3.5.1.

\section{An den österreichischen Gesetzgeber}

Empfehlung 3.2.11: Ausdrückliche Klarstellung der fehlenden Arzneimitteleigenschaft von Keimzellen zu Fortpflanzungszwecken

Dem österreichischen Gesetzgeber wird empfohlen, die fehlende Arzneimitteleigenschaft von Keimzellen zu Fortpflanzungszwecken im AMG ausdrücklich klarzustellen, etwa durch Aufnahme in die Ausnahmebestimmung des $\S 1$ Abs. 3 AMG. Keimzellen zu Fortpflanzungszwecken unterliegen den Beschränkungen des Fortpflanzungsmedizingesetzes (FMedG), des GTG und des GSG, die einen im Vergleich zum AMG über weite Strecken sachgerechteren Rechtsrahmen bieten.

Einer Verwendung hiPS-Zell-basierter Keimzellen zu Fortpflanzungszwecken steht im österreichischen Recht das Verbot des Keimbahneingriffs nach $§ 9$ Abs. 3 FMedG und $\S 64$ GTG entgegen. 


\subsection{Transplantations- und Geweberecht}

\section{Begriffsbestimmungen}

\section{An den deutschen Gesetzgeber}

Empfehlung 3.3.1: Neuformulierung der Definition der Gewebeeinrichtung in § 1 a Nr. 8 TPG

Dem deutschen Gesetzgeber wird empfohlen, die Definition der Gewebeeinrichtung in $\S 1$ a Nr. 8 TPG zur sprachlichen Vereinfachung neu zu formulieren. Von der derzeitigen, sehr weiten Begriffsbestimmung sind Entnahmeeinrichtungen mitumfasst. Dies führt dazu, dass der Gesetzgeber im TPG unterschiedliche Formulierungen für den Adressaten seiner auferlegten Rechtspflichten verwenden muss. So unterscheidet zum Beispiel § 8d TPG umständlich zwischen den Formulierungen „Gewebeeinrichtung, die Gewebe entnimmt oder untersucht" (nur Entnahmeeinrichtungen) und „eine Gewebeeinrichtung“ bzw. ,jede Gewebeeinrichtung“ (alle Gewebeeinrichtungen). Eine Neuformulierung des Begriffs „Gewebeeinrichtung“ in $\S$ 1a Nr. 8 TPG sollte zudem vor dem Hintergrund einer Vereinheitlichung der Begriffsbestimmungen in $\S 1$ TPG-Gewebeverordnung (TPG-GewV), § 20b AMG und $\S 2$ Nr. 10 und Nr. 11 Arzneimittel- und Wirkstoffherstellungsverordnung (AMWHV) erfolgen und würde so zu mehr Rechtsklarheit beitragen.

\section{Anwendungsbereich des TPG und des GSG}

\section{An den deutschen Gesetzgeber}

Empfehlung 3.3.2: Schaffung eines deutschen Gewebesicherheitsgesetzes

Dem deutschen Gesetzgeber wird empfohlen, ein Gewebesicherheitsgesetz zu schaffen. Die RL 2004/23/EG (Gewebe-RL) wurde durch das Gesetz über Qualität und Sicherheit von menschlichen Geweben und Zellen (Gewebegesetz) vom 20. Juli 2007 in deutsches Recht umgesetzt. Das Gewebegesetz führte vor allem zu Änderungen im AMG, TPG und Transfusionsgesetz (TFG).

Das Zusammenspiel von AMG und TPG im Hinblick auf den Umgang mit menschlichem Gewebe ist komplex und undurchsichtig. Ein neues Gewebesicherheitsgesetz würde mehr Transparenz und Rechtsklarheit schaffen.

Mit einem deutschen Gewebesicherheitsgesetz würde zudem die Verwendung von menschlichen Geweben (einschließlich einzelner menschlicher Zellen) von der Verwendung von menschlichen Organen (für Organe würde weiterhin das TPG gelten) rechtlich getrennt geregelt. Bereits die Gewebe-RL stellt in ihrem ErwG 9 fest, dass es „gravierende Unterschiede“ (namentlich unterschiedliche Qualitäts- und Sicherheitserfordernisse) bei der Verwendung von Organen und bei der Verwendung von Zellen und Geweben gibt, ,weshalb diese beiden Themen nicht gemeinsam in einer Richtlinie behandelt werden sollten“. Konsequenterweise schließt die Gewebe-RL deshalb nach Art. 2 Abs. 2 lit. c „Organe oder Teile von Organen, wenn sie zum gleichen Zweck wie das ganze Organ im menschlichen Körper verwendet werden sollen" von ihrem Anwendungsbereich aus.

Empfehlung 3.3.3: Klarstellung des Anwendungsbereichs des TFG für die Gewinnung von Blut und Blutbestandteilen zur Herstellung von hiPS-Zellen und 


\section{ihren Differenzierungsderivaten sowie für die Anwendung von hiPS-Zell-deri- vierten Blutprodukten}

Dem deutschen Gesetzgeber wird empfohlen, den Anwendungsbereich des TFG für die Gewinnung von Blut- und Blutbestandteilen zur Herstellung von hiPS-Zellen und ihren Differenzierungsderivaten sowie für die Anwendung von hiPS-Zell-derivierten Blutprodukten explizit klarzustellen. Es sollte insbesondere geklärt werden, ob das TFG für die Blutentnahme und die Untersuchungen der Blutzellen als Ausgangsmaterial für die Herstellung von hiPS-Zell-basierten Produkten zur Anwendung bei Menschen sowie für die Anwendung von hiPS-Zell-basierten Differenzierungsderivaten, und zwar Blutprodukten, gilt. Dem Gesetzgeber wird zudem nahegelegt, die für die Abgrenzung des Anwendungsbereichs des TPG (vgl. § 1 Abs. 3 Nr. 2 TPG) zentralen Begriffe „Blut“ und „Blutbestandteile“ in $\S 2$ TFG zu definieren. Darüber hinaus sollte die Ausnahme vom Anwendungsbereich des TFG ,autologes Blut zur Herstellung von biotechnologisch bearbeiteten Gewebeprodukten“" in § 28 TFG klarer gefasst werden. Der Gesetzgeber sollte insbesondere klarstellen, ob sich die Ausnahme nur auf die Entnahme einer geringen Menge Blut zur Vermehrung oder Aufbereitung von autologen Körperzellen bezieht (entsprechend § 20b Abs. 4 AMG) und ob alle ATMPs (und nicht „nur“ biotechnologisch bearbeitete Gewebeprodukte) von der Ausnahme erfasst sein sollen.

\subsection{Gentechnikrecht}

Die Anwendbarkeit der gentechnikrechtlichen Regelungen auf hiPS-Zellen sowie ihre Herstellung und Anwendung hängt davon $a b$, ob gewisse rechtlich festgelegte Definitionen (insbesondere von Organismen, Mikroorganismen und gentechnisch veränderten Organismen) erfüllt sind. Diese Definitionen sind allerdings teilweise sehr unklar. Es wird daher empfohlen, die Begriffsbestimmungen zu präzisieren, anzupassen bzw. teilweise neu zu formulieren, um die Rechtssicherheit im Bereich des Gentechnikrechts, insbesondere in Bezug auf neuartige Technologien wie hiPS-Zellen, zu erhöhen.

\section{Organismen und Mikroorganismen}

\section{An den europäischen Gesetzgeber}

Empfehlung 3.4.1: Legaldefinitionen der biologischen Einheit, Vermehrungsund Übertragungsfähigkeit im Zusammenhang mit dem Organismusbegriff in Art. 2 Nr. 1 RL 2001/18/EG

Art. 2 Nr. 1 RL 2001/18/EG (Freisetzungs-RL) definiert den Begriff „Organismus" und bezieht sich dabei auf biologische Einheiten sowie die Fähigkeit, sich zu vermehren oder genetisches Material zu übertragen. Dem europäischen Gesetzgeber wird empfohlen, die unklaren Begriffe der biologischen Einheit, Vermehrungsund Übertragungsfähigkeit zu definieren.

Insbesondere sollte der europäische Gesetzgeber ausdrücklich festlegen, was vom Begriff „,biologische Einheit“ im Einzelnen erfasst ist, und unter welchen Be- 
dingungen eine biologische Einheit ,fähig ist, sich zu vermehren“. Zudem sollte klargestellt werden, was unter der Fähigkeit, ,genetisches Material zu übertragen“, zu verstehen ist, insbesondere im Hinblick auf Plasmide. Hierbei ist zu klären, ob der Begriff der Übertragungsfähigkeit nur in-vitro- oder auch in-vivo-Bedingungen erfasst.

Empfehlung 3.4.2: Legaldefinitionen der mikrobiologischen Einheit, Vermehrungs- und Weitergabefähigkeit im Zusammenhang mit dem Mikroorganismusbegriff in Art. 2 lit. a RL 2009/41/EG

Art. 2 lit. a RL 2009/41/EG (System-RL) enthält eine Definition des Begriffs „Mikroorganismus“ und verwendet dabei unbestimmte Formulierungen wie ,mikrobiologische Einheit“ oder ,zur Vermehrung oder zur Weitergabe von genetischem Material fähig“. Dem europäischen Gesetzgeber wird daher empfohlen, die Begriffe der mikrobiologischen Einheit, Vermehrungs- und Weitergabefähigkeit zu definieren. Auf die Ausführungen in Empfehlung 3.4.1 wird entsprechend verwiesen.

An den deutschen Gesetzgeber

Empfehlung 3.4.3: Legaldefinitionen der biologischen Einheit, Vermehrungsund Übertragungsfähigkeit im Zusammenhang mit dem Organismusbegriff in § 3 Nr. 1 des Gentechnikgesetzes (GenTG)

Dem deutschen Gesetzgeber wird empfohlen, die Begriffe der biologischen Einheit, Vermehrungs- und Übertragungsfähigkeit für den Begriff „Organismus“ in $\S 3$ Nr. 1 GenTG zu definieren. Sollte der europäische Gesetzgeber Empfehlung 3.4.1 folgen, wird dem deutschen Gesetzgeber die Eins-zu-eins-Umsetzung der neuen Begriffsbestimmungen in der Freisetzungs-RL in nationales Recht empfohlen.

Empfehlung 3.4.4: Anpassung des Begriffs ,Mikroorganismen“ in $\$ 3 \mathrm{Nr}$. 1a GenTG an den aktuellen Stand der Technik

Dem deutschen Gesetzgeber wird empfohlen, den Begriff „Mikroorganismen“ in $\S 3$ Nr. 1a GenTG zu erneuern. Die abschließende Aufzählung von Mikroorganismen als speziellen Organismen in $\S 3$ Nr. 1a GenTG sollte an den aktuellen Stand der Technik angepasst werden. Dem Gesetzgeber wird insbesondere nahegelegt, seinen Standpunkt zu überdenken, dass Plasmide generell keine Mikroorganismen i. S. d. GenTG seien. Darüber hinaus sollte der Gesetzgeber ausdrücklich klarstellen, ob der Begriff ,tierische Zellkulturen“ in $\S 3 \mathrm{Nr}$. 1a GenTG i. S. d. naturwissenschaftlichen Sprachgebrauchs $\mathrm{zu}$ verstehen ist und deshalb auch menschliche Zellen umfasst.

\section{An den österreichischen Gesetzgeber}

Empfehlung 3.4.5: Neuformulierung des Begriffs „Organismen“ in $\$ 4$ Z. 1 GTG

§ 4 Z. 1 GTG definiert Organismen als „,ein- oder mehrzellige Lebewesen oder nichtzelluläre vermehrungsfähige biologische Einheiten einschließlich Viren, Viroide und unter natürlichen Umständen infektiöse und vermehrungsfähige Plasmide“. Diese Definition birgt erhebliche Auslegungsschwierigkeiten. Der österreichische Gesetzgeber sollte den Organismusbegriff daher neu formulieren. Insbesondere wird empfohlen, die unionsrechtlichen Vorgaben durch die explizite 
Aufnahme des Passus „oder genetisches Material zu übertragen“ in $§ 4$ Z. 1 GTG umzusetzen.

Empfehlung 3.4.6: Neuformulierung des Begriffs „Mikroorganismen“ in $\$ 4$

\section{Z. 2 GTG}

$\S 4$ Z. 2 GTG enthält eine Definition für Mikroorganismen (,,mikrobielle Organismen und kultivierte tierische und pflanzliche Zellen“), die sehr unklar ist. Es wird dem österreichischen Gesetzgeber daher geraten, den Mikroorganismusbegriff zu novellieren. Insbesondere die Tautologie „mikrobielle Organismen“ sollte durch eine aussagekräftigere Formulierung ersetzt werden. Darüber hinaus sollte der Gesetzgeber klarstellen, ob der Begriff der ,tierischen Zellen“ in Anlehnung an ein naturwissenschaftliches Verständnis auch menschliche Zellen erfasst.

\section{Gentechnisch veränderte Organismen (GVO)}

\section{An den europäischen Gesetzgeber}

Empfehlung 3.4.7: Vereinheitlichung der Sprachfassungen des Anhangs I A Teil 1 Nr. 1 RL 2001/18/EG und Anhangs I Teil A Nr. 1 RL 2009/41/EG hinsichtlich des Begriffs der Nukleinsäure- bzw. DNS-Rekombinationstechniken

Im Anhang I A Teil 1 Freisetzungs-RL und Anhang I Teil A System-RL werden bestimmte Verfahren aufgezählt, die zu einem gentechnisch veränderten Organismus (GVO) führen. Eines dieser Verfahren betrifft Rekombinationstechniken, ,bei denen durch die Insertion von Nukleinsäuremolekülen, die auf unterschiedliche Weise außerhalb eines Organismus erzeugt wurden, in Viren, bakterielle Plasmide oder andere Vektorsysteme neue Kombinationen von genetischem Material gebildet werden und diese in einen Wirtsorganismus eingebracht werden, in dem sie unter natürlichen Bedingungen nicht vorkommen, aber vermehrungsfähig sind“. Die meisten Sprachfassungen beziehen sich generell auf NukleinsäureRekombinationstechniken. Zum Beispiel sprechen die englischen Fassungen von „,recombinant nucleic acid techniques“. In anderen Sprachfassungen, wie auch in den deutschen Versionen, ist hingegen von „DNS-Rekombinationstechniken“ die Rede. Dem europäischen Gesetzgeber wird empfohlen, den Begriff der Nukleinsäure- bzw. DNS-Rekombinationstechniken in den Sprachfassungen der Freisetzungs-RL und der System-RL zu vereinheitlichen.

In diesem Zusammenhang wird dem europäischen Gesetzgeber zudem eine Klarstellung empfohlen, ob für das Vorliegen der Voraussetzungen des Anhangs I A Teil 1 Nr. 1 Freisetzungs-RL und des Anhangs I Teil A Nr. 1 System-RL bereits ein vorübergehendes (versus permanentes) Vorhandensein des genetischen Materials im Wirtsorganismus ausreicht. Ferner sollte der Gesetzgeber klarstellen, ob der Begriff ,,vermehrungsfähig“ in diesem Kontext eine dauerhafte Vermehrungsfähigkeit des eingebrachten genetischen Materials im Wirtsorganismus verlangt.

\section{Somatische Gentherapie}

\section{An den österreichischen Gesetzgeber}

Empfehlung 3.4.8: Willensbildungsprozess in Bezug auf die Definition der somatischen Gentherapie in \$ 4 Z. 24 GTG

Die Definition der somatischen Gentherapie in $\$ 4$ Z. 24 GTG und die arzneimittelrechtliche Definition des Gentherapeutikums in Anhang I Teil IV RL 2001/83/EG stimmen nicht überein. Der österreichische Gesetzgeber sollte überlegen, ob es bei 
einer somatischen Gentherapie i. S. d. Gentechnikrechts wie im Arzneimittelrecht auf einen unmittelbaren Zusammenhang zwischen therapeutischer, prophylaktischer oder diagnostischer Wirkung und eingebrachter Nukleinsäure ankommen soll und gegebenenfalls eine Änderung der Definition vornehmen.

Falls der österreichischen Gesetzgeber an der derzeitigen Definition der somatischen Gentherapie in § 4 Z. 24 GTG festhält, sollte er den Begriff ,exprimierbare Nukleinsäure" definieren. In diesem Zusammenhang sollte auch geklärt werden, ob mRNA eine exprimierbare Nukleinsäure i. S. d. § 4 Z. 24 GTG sein kann.

\section{Sonstiges}

\section{An den österreichischen Gesetzgeber}

Empfehlung 3.4.9: Korrektur des Verweises in \$2 Abs. 3 GTG

$\S 2$ Abs. 3 GTG bezieht sich hinsichtlich der Abgrenzung des Geltungsbereichs des GTG und des AMG auf Bestimmungen im Arzneimittelrecht. Um den Verweis an die geltende Fassung des AMG anzupassen, wird dem österreichischen Gesetzgeber empfohlen, die Wortfolge „Arzneimitteln im Sinne des § 1 Abs. 1 und Abs. 2 Z 1 Arzneimittelgesetz“ in § 2 Abs. 3 GTG durch „Arzneimitteln im Sinne des $§ 1$ Abs. 1 und Abs. 2 Arzneimittelgesetz“"zu ersetzen.

\subsection{Stammzellen-, Embryonenschutz- und Fortpflanzungsmedizinrecht}

\section{HiPS-Zell-basierte Keimzellen}

\section{An den deutschen Gesetzgeber}

Empfehlung 3.5.1: Schaffung eines deutschen Fortpflanzungsmedizingesetzes

Dem deutschen Gesetzgeber wird empfohlen, ein umfassendes Fortpflanzungsmedizingesetz zu schaffen. In dieses Fortpflanzungsmedizingesetz sollten insbesondere auch Regelungen zur Herstellung und Verwendung von künstlichen Keimzellen aufgenommen werden. In dem Fortpflanzungsmedizingesetz sollte der Gesetzgeber zudem die Definition des Embryos an die Begriffsbestimmung des Stammzellgesetzes anpassen und gegebenenfalls den Begriff der Totipotenz präzisieren.

Danksagung Die Autoren bedanken sich beim Bundesministerium für Bildung und Forschung für die Förderung des Verbundprojekts „ClinhiPS: Eine naturwissenschaftliche, ethische und rechtsvergleichende Analyse der klinischen Anwendung von humanen induzierten pluripotenten Stammzellen in Deutschland und Österreich“ (FKZ 01GP1602A, 01GP1602B und 01GP1602C). Diese Empfehlungen geben dabei ausschließlich die Auffassung der Autoren wieder.

\section{Literatur}

ISSCR = International Society for Stem Cell Research (2016) Guidelines for Stem Cell Research and Clinical Translation. https://www.isscr.org/docs/default-source/all-isscr-guidelines/guidelines-2016/isscrguidelines-for-stem-cell-research-and-clinical-translationd67119731dff6ddbb37cff0000940c19.pdf?sfvrsn=4. Zugegriffen am 16.01.2020 
Sara Gerke, Dipl.-Jur. Univ., M. A. Medical Ethics and Law, ist Research Fellow, Medicine, Artificial Intelligence, and Law, am Petrie-Flom Center for Health Law Policy, Biotechnology, and Bioethics at Harvard Law School in Cambridge, USA. Bis 31. März 2018 war Frau Gerke Geschäftsführerin des Instituts für Deutsches, Europäisches und Internationales Medizinrecht, Gesundheitsrecht und Bioethik der Universitäten Heidelberg und Mannheim sowie Gesamtkoordinatorin des Projekts ClinhiPS.

Dr. phil. Solveig Lena Hansen ist wissenschaftliche Mitarbeiterin am Institut für Ethik und Geschichte der Medizin in Göttingen. 2017 erhielt sie den Nachwuchspreis der Akademie für Ethik in der Medizin. Promoviert wurde sie 2016 als erste Doktorandin im Fach „Bioethik“ an der Philosophischen Fakultät der Universität Göttingen mit einer Arbeit zum reproduktiven Klonen. Andere Forschungsschwerpunkte sind ethische Aspekte von Gesundheitskommunikation, Narrative Ethik, Organtransplantation und Methodenfragen der Bioethik.

Mag. Dr. Verena Christine Blum studierte Rechtswissenschaften an der Universität Wien. Sie war von 2013 bis 2018 (mit Unterbrechung) an der Abteilung Medizinrecht des Instituts für Staatsund Verwaltungsrecht der Universität Wien tätig; zuletzt als post doc Projektmitarbeiterin im Rahmen des Projekts ClinhiPS, das sie bereits zuvor betreut hat. In ihrer Forschung beschäftigt sie sich intensiv mit ärztlichem Berufsrecht und medizinrechtlichen Werbebeschränkungen.

Dr. Stephanie Bur studierte Human- und Molekularbiologie an der Universität des Saarlandes. Während ihrer Promotion untersuchte sie die Wirkung des extrazellulären Adhäsionsproteins von Staphylococcus aureus auf die Wundheilung und Internalisierung in Hautzellen. Von 2012 bis 2018 arbeitete sie am Fraunhofer IBMT als wissenschaftliche Mitarbeiterin im Bereich der Stammzellforschung, wobei ihr Fokus darauf lag, optimierte Expansions- und Differenzierungsmethoden in Suspensions-Bioreaktor-Systemen zu entwickeln.

Clemens Heyder, M.A. M.mel. studierte Philosophie und Geschichte an den Universitäten Leipzig, Basel und Halle, an der er den Masterstudiengang Medizin-Ethik-Recht absolvierte. Zur Zeit promoviert er über die ethischen Aspekte der Eizellspende und ist als Dozent in der Erwachsenenbildung tätig. Während seiner Tätigkeit am Translationszentrum für regenerative Medizin Leipzig sowie am Institut für Ethik und Geschichte der Medizin Göttingen entwickelte er ein Interesse für die Forschungsethik. Weitere Forschungsinteressen: Ethik der Reproduktionsmedizin, Autonomie, normative Ethik.

Univ.-Prof. DDr. Christian Kopetzki studierte Rechtswissenschaften (Dr. iur. 1979) und Medizin (Dr. med. 1984) an der Universität Wien. 1995 habilitierte er sich in den Fächern Medizinrecht, Verfassungsrecht und Verwaltungsrecht. Seit 2002 ist er Universitätsprofessor für Medizinrecht am Institut für Staats- und Verwaltungsrecht der Universität Wien. Er ist Autor zahlreicher Bücher und Aufsätze (u. a. zum Gentechnik-, Biotechnologie- und Fortpflanzungsmedizinrecht, zur Stammzellforschung und zu Grundrechten im Bereich der Biomedizin).

Dr. Ina Meiser studierte Bioinformatik (B.Sc.) und Biotechnologie (M.Sc.) an der Universität des Saarlandes und absolvierte 2014 ihre Dissertation zum Thema „Untersuchungen zur Präparation komplexer Zellsysteme im Kontext neuer Therapien “ am Fraunhofer IBMT. Als wissenschaftliche Mitarbeiterin arbeitet sie am IBMT an Automatisierungsstrategien in der Zellkultur sowie an neuartigen Technologien zur anwendungsorientierten Kryokonservierung, insbesondere mit humanen Stammzellen, und leitet seit 2016 die Arbeitsgruppe Kryobiotechnologie.

Dr. Julia C. Neubauer Diplom in Biologie, Ludwig-Maximilians-Universität Würzburg. 2012 Dissertation, Universität des Saarlandes. Seit 2007 am Fraunhofer-Institut für Biomedizinische Technik (IBMT) im Saarland, seit 2012 dort Arbeitsgruppenleiterin, seit 2014 Abteilungsleiterin mit Schwerpunkt auf der Entwicklung von Automatisierungsstrategien für Stammzellanwendun- 
gen und Kryokonservierungstechnologien für therapeutisch relevante Zellen. Seit 2017 Geschäftsführerin des Fraunhofer-Projektzentrums für Stammzellprozesstechnik in Würzburg.

Mag. Danielle Noe studiert seit 2013 Rechtswissenschaften an der Universität Wien (Mag. iur. 2017). Seit 2016 ist sie in der Abteilung Medizinrecht des Instituts für Staats- und Verwal tungsrecht der Universität Wien beschäftigt. Zunächst war sie als Projektmitarbeiterin im Rahmen des Projekts ClinhiPS, seit 2017 ist sie als wissenschaftliche Mitarbeiterin (prae doc) tätig. Der Schwerpunkt ihrer Forschung liegt im Biotechnologierecht, insbesondere im Bereich der medizinrechtlichen Regulierung neuartiger Therapiemethoden.

Mag. Dr. Claudia Steinböck (vormals Zeinhofer) studierte Rechtswissenschaften an der Universität Wien (Mag. iur 2003, Dr. iur 2007). Ihre Dissertation zum Arzneimittelbegriff wurde mit dem Wolf Theiss Award 2007 ausgezeichnet. Sie ist (mit Unterbrechung) seit 2003 als wissenschaftliche Mitarbeiterin an der Abteilung Medizinrecht des Instituts für Staats- und Verwaltungsrecht der Universität Wien tätig und am Projekt ClinhiPS beteiligt. Ihre Forschungs- und Publikationsschwerpunkte liegen im Arzneimittel- und Gewebesicherheitsrecht.

Prof. Dr. Claudia Wiesemann ist Direktorin des Instituts für Ethik und Geschichte der Medizin an der Universitätsmedizin Göttingen und stellvertretende Vorsitzende des Deutschen Ethikrats. Von 2002-2012 war sie Mitglied der Zentralen Ethik-Kommission für Stammzellenforschung am Robert-Koch-Institut. Sie leitete das europäische Forschungsprojekt Tiss.Eu (zus. mit C. Lenk) zu Ethik und Recht von Forschung mit menschlichem Gewebe. Weitere Schwerpunkte sind die Ethik der Fortpflanzungsmedizin und Kinderrechte in der Medizin.

Prof. Dr. Heiko Zimmermann Studium der Physik an der Universität Würzburg und der Humboldt-Universität zu Berlin. 2001 Promotion in experimenteller Biophysik an der Humboldt-Universität zu Berlin. 2004 Juniorprofessur an der Universität des Saarlandes, 2008 W3-Professur „Molekulare \& zelluläre Biotechnologie/Nanotechnologie“ (Universität des Saarlandes). Parallel seit 2001 Leitungsfunktionen am Fraunhofer-Institut für Biomedizinische Technik IBMT. Seit 2012 Institutsleiter am IBMT.

Prof. Dr. Jochen Taupitz ist Inhaber des Lehrstuhls für Bürgerliches Recht, Zivilprozessrecht, internationales Privatrecht und Rechtsvergleichung an der Universität Mannheim und Geschäftsführender Direktor des Instituts für Deutsches, Europäisches und Internationales Medizinrecht, Gesundheitsrecht und Bioethik der Universitäten Heidelberg und Mannheim. Er ist u. a. Vorsitzender der Zentralen Ethikkommission bei der Bundesärztekammer sowie Mitglied der Nationalen Akademie der Wissenschaften Leopoldina und der Academia Europaea.

Open Access Dieses Kapitel wird unter der Creative Commons Namensnennung 4.0 International Lizenz (http://creativecommons.org/licenses/by/4.0/deed.de) veröffentlicht, welche die Nutzung, Vervielfältigung, Bearbeitung, Verbreitung und Wiedergabe in jeglichem Medium und Format erlaubt, sofern Sie den/die ursprünglichen Autor(en) und die Quelle ordnungsgemäß nennen, einen Link zur Creative Commons Lizenz beifügen und angeben, ob Änderungen vorgenommen wurden.

Die in diesem Kapitel enthaltenen Bilder und sonstiges Drittmaterial unterliegen ebenfalls der genannten Creative Commons Lizenz, sofern sich aus der Abbildungslegende nichts anderes ergibt. Sofern das betreffende Material nicht unter der genannten Creative Commons Lizenz steht und die betreffende Handlung nicht nach gesetzlichen Vorschriften erlaubt ist, ist für die oben aufgeführten Weiterverwendungen des Materials die Einwilligung des jeweiligen Rechteinhabers einzuholen. 